\title{
A Metabonomic Approach to Analyze the Dexamethasone-Induced Cleft Palate in Mice
}

\author{
Jinglin Zhou, ${ }^{1}$ Bin Xu, ${ }^{1}$ Bing Shi, ${ }^{1}$ Jing Huang, ${ }^{2}$ Wei He, ${ }^{1}$ Shengjun Lu, ${ }^{1}$ Junjun Lu, ${ }^{1}$ \\ Liying Xiao, ${ }^{1}$ and $\mathrm{Wei} \mathrm{Li}^{1,3}$
}

\author{
${ }^{1}$ State Key Laboratory of Oral Diseases, Sichuan University, Chengdu 610041, China \\ ${ }^{2}$ West China College of Pharmacy, Sichuan University, Chengdu 610041, China \\ ${ }^{3}$ State Key Laboratory of Stomatology, Sichuan University, No.14 Sec 3 Renminnan Road, Chengdu 610041, China
}

Correspondence should be addressed to Wei Li, leewei2000@sina.com

Received 6 February 2010; Revised 3 April 2010; Accepted 8 June 2010

Academic Editor: Mika Ala-Korpela

Copyright () 2011 Jinglin Zhou et al. This is an open access article distributed under the Creative Commons Attribution License, which permits unrestricted use, distribution, and reproduction in any medium, provided the original work is properly cited.

\begin{abstract}
Mice models are an important way to understand the relation between the fetus with cleft palate and changes of maternal biofluid. This paper aims to develop a metabonomics approach to analyze dexamethasone-induced cleft palate in pregnant C57BL/6J mice and to study the relationship between the change of endogenous small molecular metabolites in maternal plasma and the incidence of cleft palate. To do so, pregnant mice were randomly divided into two groups. The one group was injected with dexamethasone. On E17.5th day, the incident rates of cleft palate from embryos in two groups were calculated. The ${ }^{1} \mathrm{H}-\mathrm{NMR}$ spectra from the metabolites in plasma in two groups was collected at same time. Then the data were analyzed using metabonomics methods (PCA and SIMCA). The results showed that the data from the two groups displayed distinctive characters, and the incidence of cleft palate were significantly different $(P<.005)$. To conclude, this study demonstrates that the metabonomics approach is a powerful and effective method in detecting the abnormal metabolites from mother in the earlier period of embryos, and supports the idea that a change from dexamethasone induced in maternal metabolites plays an important role in the incidence of cleft palate.
\end{abstract}

\section{Introduction}

Congenital cleft lip and palate (CLP) is the most common birth defect in humans. The etiology is complicated, and it involves genetic and environmental factors [1,2]. Maternal condition during pregnancy also appears to play an important role [3]. Smoking [4-6], over intake of vitamin A [7, 8], and deficiency in folic acid and Bs may increase risk for oral clefts [9-12]. In addition, drug-induced (corticosteroid) teratogenesis has also received some attention [13-16]. Most studies above are based on epidemiological investigation. As a useful model, experiments of mouse development are used to assess the mechanism of palate defects in fetuses resulting from exposure to the risk factors. Current strategies to study orofacial defects focus on the related genotype and transcription factors $[17,18]$. However, the capability of maternal detoxification during orofacial development is important for normal palate formation. As a result, a fetus lacking genes associated with cleft palates still has high risk of defect if maternal detoxification is insufficient. $[2,19]$

Metabonomics, based on the NMR spectroscopic and multivariate statistics, can be useful for the description and recognition of the dynamic multivariate metabolic response of an organism to a pathological event or genetic modification. The ${ }^{1} \mathrm{H}-\mathrm{NMR}$ spectra of the biofluids from an organism contain a significant amount of useful metabolic information. Application of automated data reduction algorithms and chemometric analysis, which is called pattern recognition analysis (PR), can be competent for the description and recognition of the dynamic multivariate metabolism. It is included two major approaches, one is termed "unsupervised", which could be used to not only reduce the complex and volume data to a suitable level but also screen for the outlier and examine condition of the clusters, including Principal Component Analysis (PCA), Nonlinear Mapping (NLM) and Hierarchical Cluster Analysis (HCA). The other is termed "supervised" that 


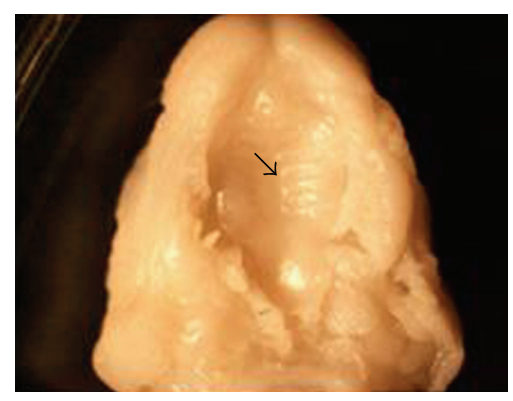

(a)

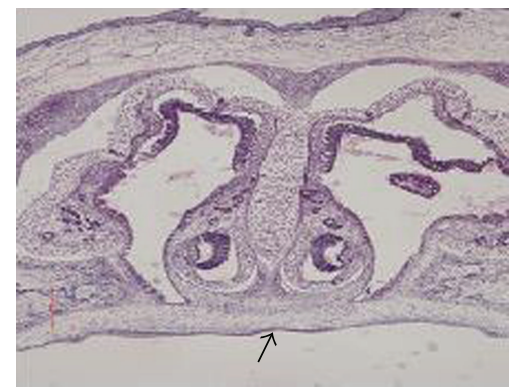

(c)

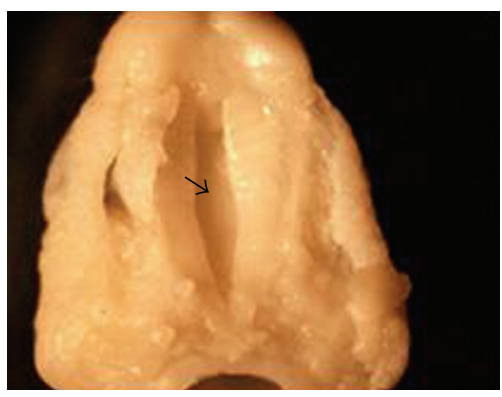

(b)

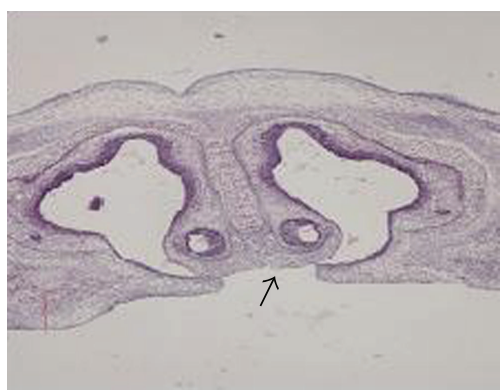

(d)

FIGURE 1: Photos of fusion or lack of fusion by embryonic palatal shelves. The fusion situation of embryonic palatal shelves was observed and photographed. (a) Fused embryonic palatal shelf. (b) Nonfused embryonic palatal shelf. (c) Fused embryonic palatal shelf in histological section. (d) Nonfused embryonic palatal shelf in histological section.

means, make a mathematical model by a training set of known class from the samples and then draw the estimated or predictive model by independent validation set. There are many methods such as Soft Independent Modeling of Class Analogy (SIMCA), Partial Least Squares (PLS), Linear discriminant analysis (LDA), K-nearest neighbor analysis (KNN), and Bayesian methods. These approaches have been successfully applied to study diseases and toxic processes [20-27]. Principal component analysis (PCA) as a bilinear decomposition method is one of the most easy and efficient approaches to analyze the NMR data, A PCA model can trustily display a summary of all samples in the data table [27]. Combination with using a prediction model, such as SIMCA, can be constructed to analyze the unknown samples [28-30].

Development of the mammalian palate involves a number of critical steps: growth, elevation, contact, and fusion (the medial edge epithelium disappearance). Any problem arising from these steps can lead to the incidence of cleft palate. DEX, one of the glucocorticoids (GC), can penetrate the blood-placental barrier and bind to GC receptor (GR) in the cytoplasm, and can depress the ability of palatal mesenchymal proliferation. Since the palate is smaller, development of bilateral palates is interrupted, which results in the cleft [31-34]. We hypothesized that the metabonomic method could provide the framework for the studies on the consequences of maternal environmental changes during pregnancy and for illustrating the relationship between the changes of maternal environment and development of palates. To test this hypothesis, we studied the plasma samples from pregnant C57BL/6J mice, which were induced with dexamethasone (DEX) to trigger cleft palate formation in the fetuses. The analyses demonstrated the relationship between the change in endogenous small molecular metabolites of maternal plasma and the incidence of cleft palate (CP) in fetuses.

\section{Materials and Methods}

2.1. Animal Handling and Dosing. All animal experimentation was approved by the Animal Research Committee of the West China College of Stomatology (Sichuan University). C57BL/6J (C57) mice, about 8 weeks of age, were obtained from the Laboratory Animal Center of Sichuan University. All mice were reared in plastic cages $(28 \mathrm{~cm} \times 16 \mathrm{~cm} \times$ $12 \mathrm{~cm}$ ), under a 12/12-hour light/dark cycle. The room was maintained at a controlled ambient temperature of 20$27^{\circ} \mathrm{C}$ with $40 \%-70 \%$ relative humidity. A commercial diet (Laboratory Animal Center of Sichuan University, China) and tap water were fed ad libitum. Mice were allowed to acclimatize for 48 hours prior to mating. Two virgin females and one male were placed overnight in a cage and checked on following morning for the copulatory plug of female mice. Females with copulation plugs were weighed immediately and the date was designated to Embryonic day $0\left(E_{0}\right)$.

Pregnant mice (42) were randomly divided into two groups (the experimental group and the control groupeach group 21 mice). From the 10th day to the 12 th day of pregnancy (From $E_{10}$ to $E_{12}$ ), the mice in the experimental group were intraperitoneally injected daily with Dexamethasone (DEX dexamethasone sodium phosphate injection, Tianjing China) at $6 \mathrm{mg} / \mathrm{kgand}$ while the others in the control group 


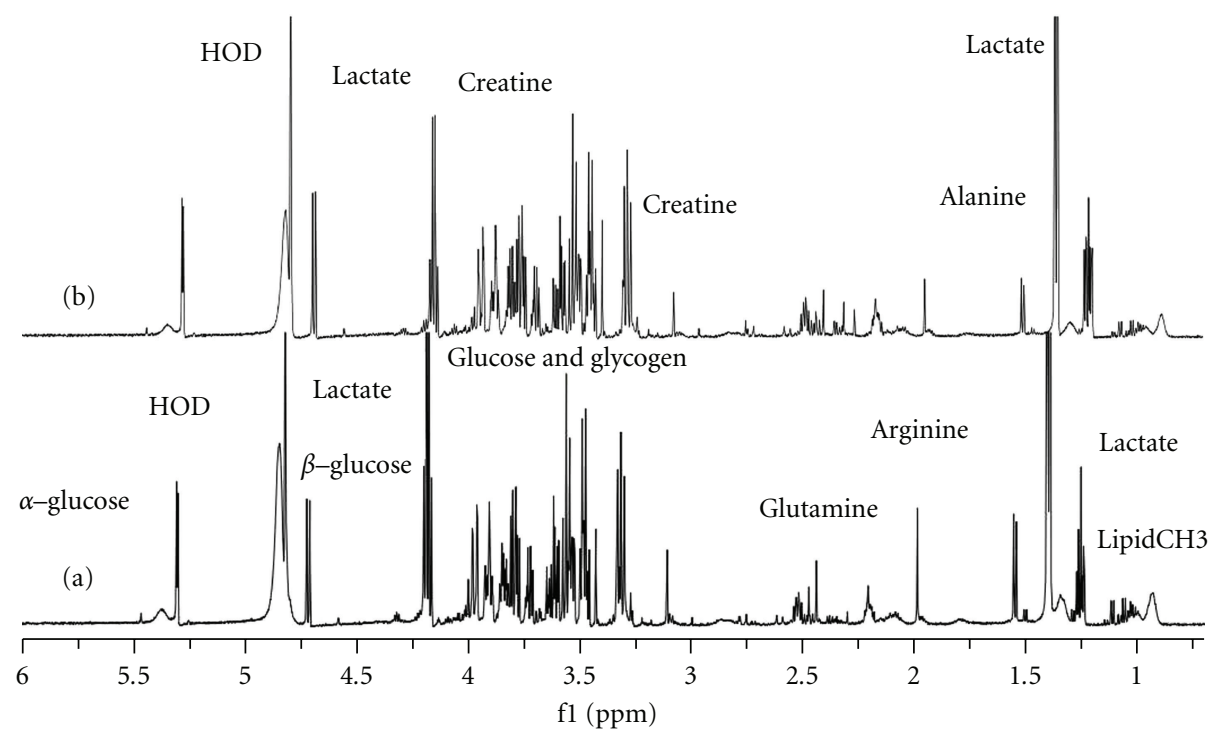

FIGURE 2: $600 \mathrm{MHz}{ }^{1} \mathrm{H}-\mathrm{NMR}$ spectra of plasma samples from the pregnant mice in two groups: "a" showed the spectrum from one sample in the control group; "b" showed the spectrum from one sample in the DEX group.

with isotonic sodium chloride $(0.9 \% \mathrm{NaCl})$. The dose levels were based on the literature report [35].

2.2. Sample Collection. On the day of $\mathrm{E}_{17.5}$, all the pregnant mice were sacrificed and weighed. The peripheral blood was placed in a lithium heparin tube (Vacuette Austria) immediately. Within 2 hours of the collection, the erythrocytes were separated by centrifugation $\left(3000 \times \mathrm{g}\right.$ at $4^{\circ} \mathrm{C}$ or $\left.10 \mathrm{~min}\right)$ to pick up the biofluid top of the plasma for analysis. All the plasma samples were stored at $-80^{\circ} \mathrm{C}$ before the ${ }^{1} \mathrm{H}$ NMR analysis. At the same time, a stereomicroscope was used to determine the number of live fetuses and gross malformations.

2.3. Statistical Analysis of the Incidence of Cleft Palate. After removal of the mandibles, embryonic palates were calculated using stereomicroscope $(10 \times)$ by morphologic in two groups. Target palates were processed into paraffin wax. Histological sections of embryonic palates were cut and stained with haematoxylin and eosin ( $\mathrm{HE})$. The results were photographed by a Leica photographic system (Germany). The numbers of CP in two groups were analyzed to detect the different incidence rates by using chi-square test and variance-based statistical algorithm (SPSS 10.0).

2.4. Preparation of Plasma Samples for ${ }^{1} H$-NMR Spectroscopy. The ${ }^{1} \mathrm{H}$-NMR spectra were obtained immediately after each sample was thawed at $300 \mathrm{~K}$ and then kept at the same temperature in a Bruker Avance II 600 spectrometer (Bruker Biospin, Germany), which was operating at $600.13 \mathrm{MHz}$ with a 5-mm PATXI probe. In order to obtain a deuterium lock signal for the NMR spectrometry, $200 \mu \mathrm{l}$ aliquots of all the plasma were diluted up to $500 \mu \mathrm{l}$ with $300 \mu \mathrm{l}$ of Deuterium oxide $\left(\mathrm{D}_{2} \mathrm{O}\right)$.
2.5. Acquisition of ${ }^{1} H-N M R$ Spectra of Plasma. The spectrum was obtained by using two different pulse sequences: one is selective presaturation pulse sequence (Bruker Biospin, Germany) for water suppression (located in $\delta 4.8 \mathrm{ppm}$ ) and other is CPMGPR1D pulse sequence (Bruker Biospin, Germany). The later one, which was used to attenuate the broad protein signal in the plasma, is a modification of the Carr-Purcell-Meiboom-Gill pulse sequence (CPMGpulse, Bruker Biospin Germany) to suppress the residual water signal. For each sample, 1D ${ }^{1} \mathrm{H}-\mathrm{NMR}$ spectrum was collected with $64 \mathrm{~K}$ data points, 64 scans and 15 ppm spectral width. Other acquisition parameters were $5 \mathrm{~s}$ relaxation delay, 8 dummy scans, $400 \mu$ s fixed echo time to allow the elimination of J-mod, and 400 CPMG loops for $\mathrm{T}_{2}$ filter. The phase and the baseline of all acquired NMR spectra were manually calibrated, and the chemical shifts corrected by the reference of the lactate doublet at $\delta 1.32$ [36] with TopSpin 1.3 (Bruker Biospin, Germany).

2.6. Reduction of the NMR Spectral Data. All signals of the 42 samples' were located in the range of $\delta 0-7 \mathrm{ppm}$ in spectral region and have no visible resonance peak after $\delta 7 \mathrm{ppm}$. Using MestReC (version 4.8.1.1, Spain), each ${ }^{1} \mathrm{H}-\mathrm{NMR}$ spectrum was divided with $\delta 0.04 \mathrm{ppm}$ width into 162 contiguous segments and integrated from the rang of $\delta 0-7 \mathrm{ppm}[37,38]$. The region of the spectrum $(\delta 5.0-4.5 \mathrm{ppm})$ was removed to exclude the influence of the water signal. The result was set up to a kind of $2 \mathrm{D}$ matrix $(n \times d)$, “ $n$ " representing the 42 samples, " $d$ " meaning 162 contiguous segments. The matrix data were normalized to the unit area with the appropriate weighting coefficients in Excel (Microsoft USA) and then exported into the SIMCA-P software package (version11.0, Umetrics AB, Umeå, Sweden). The average value of each variable was calculated and subtracted from the data. 


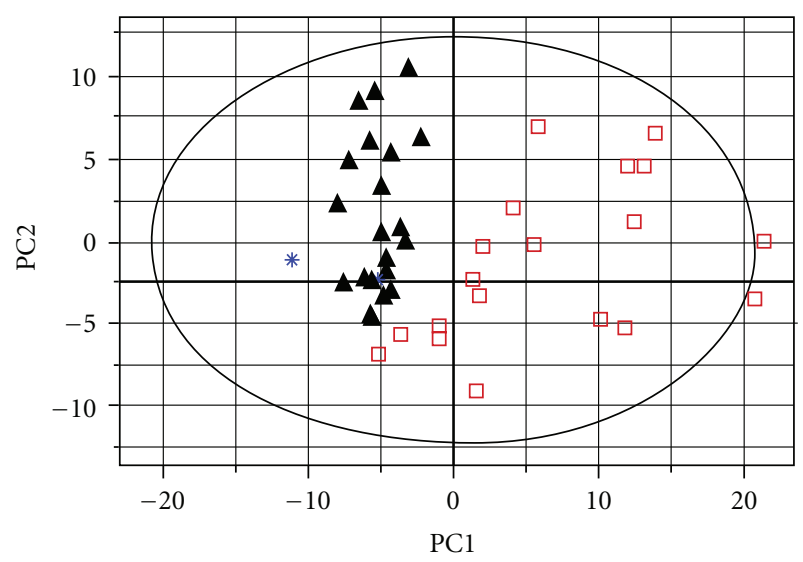

Figure 3: The plot of PCA analysis from two groups. “॥” showed the data from pregnant mice with CP fetuses in DEX group; “ $\square$ ” showed the data from pregnant mice with fusion fetuses in control group. “ $*$ ” showed the pregnant mice including CP fetuses in the control group.

TABLE 1: Comparison of the incidence of cleft palate in the embryos between two groups.

\begin{tabular}{lcccc}
\hline & Non-Fusion & Fusion & Total & Incidence of non-fusion (\%) \\
\hline Control group & 3 & 103 & 106 & 2.83 \\
DEX group & 27 & 96 & 123 & $21.95^{*}$ \\
Total & 30 & 199 & 229 & 13.10 \\
\hline
\end{tabular}

* Significant difference with respect to control $\left(\chi^{2}=18.285, P<.005\right)$.

2.7. Statistical Analysis of the Data from NMR Spectra. After the average value being calculated from each variable and subtracted from the data, PCA was applied to the meancentered data to detect the differences among the samples. SIMCA was performed to validate the results. Firstly, separate PC models were built for the training sets of 10 samples from DEX group with CP fetuses and 10 samples including only normal fetus of control group and were defined two distances against boundaries (cross-validation) by the $95 \%$ confidence interval. As a typically visualizing method, the Cooman's plot was often used to display the result [39]. SIMCA formulated a PC model for each distinct class and estimated its performance. Secondly, the procedure was repeated for all samples (the test sets) in turn, to predict and assess whether samples were separated into two classes. Finally, the result of the Cooman's plot illustrated the visible separation of two groups. Each step was repeated five times in order to keep the validation set.

\section{Results}

3.1. The Statistics of the Incidence of Cleft Palate in Two Groups. Forty-two C57BL/6J pregnant mice were examined, and 229 fetuses were collected, including 123 fetuses from DEX treatment group and 106 fetuses from the control group (Table 1). Each pregnant mouse had multiple fetuses. There was at least one CP fetus in each pregnant mouse in DEX treatment group (named DEX group), while there were $3 \mathrm{CP}$ fetuses in two pregnant mice of the control group (named the control group with $\mathrm{CP}$ ). The incidence of CP in the DEX treatment group was $21.95 \%$, significantly different from the control group $(P<.005)$. The character of morphology and histology were recorded (Figure 1). "a" and "c" show fusion of palate shelves, while " $b$ " and " $d$ " show a failure of the palate shelves to fuse.

3.2. ${ }^{1} \mathrm{H}$-NMR Spectra from Two Groups. The ${ }^{1} \mathrm{H}-\mathrm{NMR}$ spectrum of the plasma from pregnant mice revealed great complexity and significant information about the biofluid (Figure 2). The chemical shift of $\delta 4.8$ was the water signal. The regions of the significant metabolite signals usually ranged $\delta 0-4.5 \mathrm{ppm}$, including alkene ( $\delta 4-5.5 \mathrm{ppm})$, alkyne ( $\delta$ 1.8-3.5 ppm), and the aliphatic series ( $\delta 0-2.8 \mathrm{ppm})$. Articles of the locations of different chemical groups and some low-molecular-weight metabolites had already been published [36, 40]. To detect the difference between the two groups, special software was used in the chemometric analysis.

3.3. PCA of the Plasma in the DEX Group and the Control Group. The matrix data from the ${ }^{1} \mathrm{H}-\mathrm{NMR}$ spectra were exported into the SIMCA-P software and processed by PCA. The new principal component (PC) variables were created (6 new contributories of the PCs), which accounted for $84.8 \%$ of the original data and each PC was orthogonal with all the other PCs. The result of PCA could be shown (Figure 3) as the clustering of the individual dataset defined by the 95\% Hotelling's T2 Limit. Except for two control samples ( $*$ : the blue star), all the 21 DEX samples ( $\boldsymbol{\Delta}$ : the black triangles) and 19 control samples with fusion fetuses ( $\square$ : the open squares), obviously, were separated well along the first principal component. It indicated that the major difference between the two groups occurred in the first two principal 


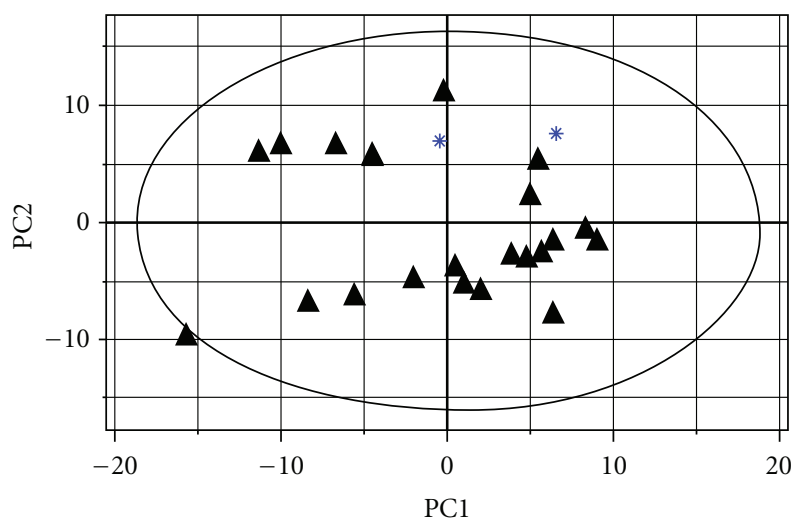

(a)

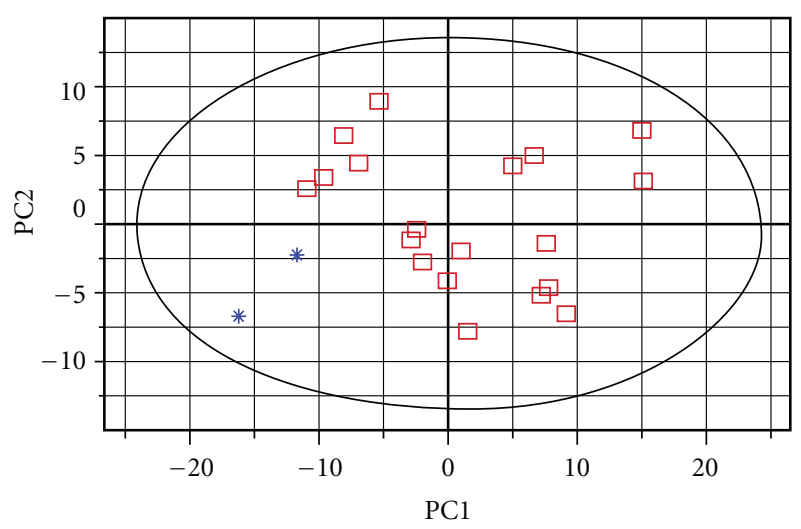

(b)

FIGURE 4: The plots of PCA analysis for two control pregnant mice including CP fetuses in different groups "a" showed the PCA analysis between two outliers from the control group and the data of pregnant mice with CP fetuses in DEX group; "b" showed the PCA analysis between two outliers from the control group and the data of pregnant mice with no-CP fetuses in control group.

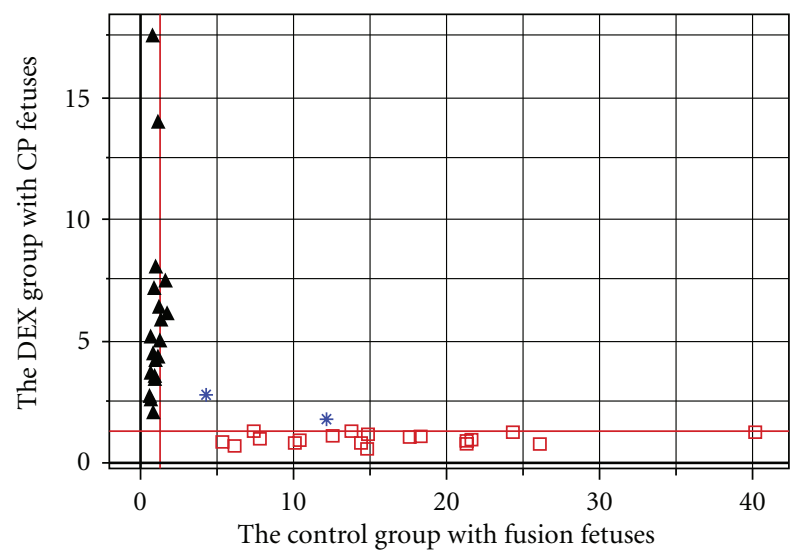

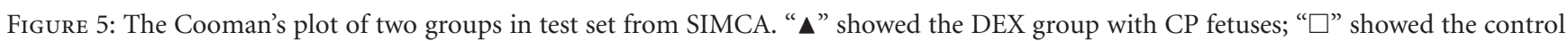
group with fusion fetuses; “*” showed two outlier samples (pregnant mice with CP fetuses in control group); the red lines indicate the D-Crit (0.05) level; two groups were located in two different spaces; the outliers not belong to either group.

components. The detailed different biochemical changes were informed by the loadings (Table 2). In order to detect the outliers, the two outliers as one group to be detected with Dex group and control group respectively. PCAs were repeated twice again using different groups (Figure 4). The result indicated that the two outliers with $\mathrm{CP}$ fetuses have some extent different from the control group, while mixed in the Dex-injected group because of CP fetuses.

\subsection{SIMCA of the Plasma in the DEX Group and the Control} Group. The result of SIMCA was shown in the Cooman's plot (Figure 5). All the samples had been tested with an independent model made of train set. The condition of samples can be classified in a pair of distances against boundaries (red lines) for non-fusion data in DEX group and fusion data in control group. The plot contained four regions separated by $95 \%$ confidence limits in a $2 \mathrm{D}$ graph in which samples below the horizontal line belonged to the control group with fusion fetuses (95\%), while samples in the left region of the vertical line belonged to DEX group with $\mathrm{CP}$ fetuses (95\%). The data from control group with CP fetuses (the outliers) neither belongs to DEX group nor belongs to control group. It demonstrated that plasma classes from pregnant mice with CP fetuses and normal control did not share the same space. Therefore, this model should be able to predict whether maternal environment has been affected by DEX or not.

\section{Discussion}

In the normal mouse embryo, palate shelves grow and elevate into a horizontal position by embryonic day 14 (E14). By the day of E17, the process of fusion has completely finished. We chose to collect samples on E17.5 for the following reasons. It was convenient to use morphology for embryo analysis, and it was easy to display the change of maternal metabolism without the influence of DEX [41]. Furthermore, another study also proved that the spectra of plasma from Dex-injected and control male mice showed no significant difference during different time frames 
TABLE 2: the main metabolites changes that were partially responsible for discriminating Dex group from control using PCA.

\begin{tabular}{llc}
\hline${ }^{1} \mathrm{H}$ bucket region $(\delta)$ & metabolites & Changes in Dex group \\
\hline $1.32,4.12$ & Lactate & $\uparrow^{*}$ \\
1.36 & unknown & $\uparrow$ \\
1.48 & Alanine $\mathrm{CH} 3$ & $\uparrow$ \\
1.72 & Arginine $(\gamma-\mathrm{H})$ & $\downarrow$ \\
1.92 & Arginine $(\beta-\mathrm{H})$ & $\uparrow$ \\
2.02 & Acetate & $\uparrow$ \\
2.04 & Lipid $\mathrm{CH}_{2} \mathrm{CH}_{2} \mathrm{CH}=\mathrm{CH}$ & $\uparrow$ \\
2.12 & Glutamine & $\downarrow$ \\
2.28 & Valine $(\beta-\mathrm{H})$ & $\uparrow$ \\
2.36 & Glutamate & $\uparrow$ \\
2.4801 & unknown & $\downarrow$ \\
2.5201 & Lipid $-\mathrm{C}=\mathrm{C}-\mathrm{CH}-\mathrm{C}=\mathrm{C}$ & $\downarrow$ \\
2.8 & Aspartic acid $(\beta-\mathrm{H})$ & $\uparrow$ \\
3.24 & Choline $\mathrm{N}(\mathrm{CH} 3) 3+$ & $\downarrow$ \\
3.64 & Valine $(\alpha-\mathrm{H})$ & $\uparrow$ \\
3.92 & Creatine & $\downarrow$ \\
4.04 & myo-inositol &
\end{tabular}

$* \uparrow$, relative increase in signal by loading plot.

(2 hours, 1 day, 2 days, 3 days, and 7 days). The PCAs also show that all data from different time frames were a mixture together, which accounted for $89.9 \%$ from the original data (supplement).

Different murine strains also have different susceptibilities to the DEX-induced cleft palate. The incidence in our model was in accord with previous work [42]. Some studies indicate [43-45] that the incidence of cleft palate may be closely related to high maternal concentration of plasma homocysteine or lower activity of the glucocorticoids prereceptor metabolizing enzyme $11 \beta$-hydroxysteroid dehydrogenase type 2 (11 $\beta 2$ HSD2) in placental trophoblastic cells. However, the exact mechanisms by which interaction between maternal environment and DEX causes teratogenic effects are poorly understood. NMR spectroscopy-based metabonomic approach offers a unique opportunity to focus on the relationship between genotype and phenotype and is especially suited to uncover changes in drug-induced metabolites [46, 47]. In this study, each mother at least has one CP fetus in DEX-injected group. Some changes of maternal metabolites informed us about the abnormality of the embryo. Therefore, the result from two groups displayed the different metabolites between CP fetus and fusion one. From the spectra of ${ }^{1} \mathrm{H}-\mathrm{NMR}$ (Figure 2), we can see clearly some differences between the samples of the two groups within the range of chemical shift from $\delta 1.8$ to $\delta 3.5 \mathrm{ppm}$ and the range of glucose and amino acid $\mathrm{CH}$. The PCA plots showed good separation of the two groups except for two control samples (Figure 3), which matched the results from morphologic analysis identifying three cleft palate fetuses from two different individual mice in the control group. In order to analyze the outlier from the control group, the two outliers as a group were analyzed again. The result also show these two samples were closely blended in the DEX group (Figure 4(a)), while far away from the controls' (Figure 4(b)). Some biochemical changes such as decrease of Arginine (1.72, $1.92 \mathrm{ppm})$, increase of Alanine (1.48 ppm), N-acetyl glycoprotein (2.12 ppm), Choline (3.24 ppm), and Creatine (3.92 ppm) may response to the change of methyl group metabolism in maternal environment. The key donor of methylation is S-adenosyl methionine (SAM) which comes from methionine. Arginine and Creatine may increase ATP to supply the circle from methionine to homocysteine [48]. Normally, the incidence of cleft palate in C57BL/6J natural mice is zero. The reason may be that we injected isotonic sodium chloride $(0.9 \% \mathrm{NaCl})$ in control samples in order to keep the same stimulating circumstance as in the DEX group. After SIMCA analysis, the result of the Cooman's plot revealed a good fit model to detect the NMR data and separate the CP group from the controls by using a test set of $F$-statistic. Both plots clearly showed a relationship between the fetus' cleft palate and the plasma metabolic profile of the mother. In conclusion, this study provides evidence that metabonomic method is sufficiently sensitive to detect small differences in the composition of maternal biofluids and may be helpful for identifying biomarkers of teratogenesis.

\section{Conflict of Interest Statement}

The authors declare no conflict of interests.

$\begin{array}{ll}\text { Abbreviations } \\ \text { PRA: } & \text { Pattern recognition analysis } \\ \text { DEX: } & \text { Dexamethasone } \\ \text { HE: } & \text { Haematoxylin and eosin } \\ \text { CPMG: } & \text { Carr-Purcell-Meiboom-Gill pulse sequence } \\ \text { PCA: } & \text { The principal component analysis } \\ \text { SIMCA: } & \text { Soft independent modeling of class analogy } \\ \text { GC: } & \text { Glucocorticoids } \\ \text { GR: } & \text { GC receptor } \\ \text { CP: } & \text { Cleft palate. }\end{array}$

\section{Acknowledgments}

This study was supported by Grants from the Key Research Project of the Ministry of Education in China (no. 307022), the National Natural Science Foundation of China (no. 30530730) and the Youth Teacher Science Foundation of Sichuan University (no. 2009SCU11162). The authors are grateful to Dr. Shan $\mathrm{Lu}, \mathrm{PhD}$ and Chapin Rodriguez, PhD for their useful comments.

\section{References}

[1] A. Jugessur and J. C. Murray, "Orofacial clefting: recent insights into a complex trait," Current Opinion in Genetics and Development, vol. 15, no. 3, pp. 270-278, 2005.

[2] A. C. Lidral, L. M. Moreno, and S. A. Bullard, "Genetic factors and orofacial clefting," Seminars in Orthodontics, vol. 14, no. 2, pp. 103-114, 2008. 
[3] J. S. Zeiger and T. H. Beaty, "Is there a relationship between risk factors for oral clefts?” Teratology, vol. 66, no. 5, pp. 205208, 2002.

[4] K. Källén, "Maternal smoking and orofacial clefts," Cleft Palate-Craniofacial Journal, vol. 34, no. 1, pp. 11-16, 1997.

[5] K. C. Chung, C. P. Kowalski, H. M. Kim, and S. R. Buchman, "Maternal cigarette smoking during pregnancy and the risk of having a child with cleft lip/palate," Plastic and Reconstructive Surgery, vol. 105, no. 2, pp. 485-491, 2000.

[6] J. Little, A. Cardy, and R. G. Munger, "Tobacco smoking and oral clefts: a meta-analysis," Bulletin of the World Health Organization, vol. 82, no. 3, pp. 213-218, 2004.

[7] R. H. Finnell, G. M. Shaw, E. J. Lammer, K. L. Brandl, S. L. Carmichael, and T. H. Rosenquist, "Gene-nutrient interactions: importance of folates and retinoids during early embryogenesis," Toxicology and Applied Pharmacology, vol. 198, no. 2, pp. 75-85, 2004.

[8] R. G. Munger, "Maternal nutrition and oral clefts," in Cleft Lip and Palate: From Origin to Treatment, D. F. E. Wyszynski, Ed., pp. 170-192, Oxford University Press, Oxford, UK, 2002.

[9] G. M. Shaw, S. L. Carmichael, C. Laurent, and S. A. Rasmussen, "Maternal nutrient intakes and risk of orofacial clefts," Epidemiology, vol. 17, no. 3, pp. 285-291, 2006.

[10] G. M. Shaw, E. J. Lammer, C. R. Wasserman, C. D. O’Malley, and M. M. Tolarova, "Risks of orofacial clefts in children born to women using multivitamins containing folic acid periconceptionally," Lancet, vol. 346, no. 8972, pp. 393-396, 1995.

[11] L. D. Botto, R. S. Olney, and J. D. Erickson, "Vitamin supplements and the risk for congenital anomalies other than neural tube defects," American Journal of Medical Genetics, vol. 125, no. 1, pp. 12-21, 2004.

[12] I. P. C. Krapels, I. A. L. M. Van Rooij, M. C. Ocké, C. E. West, C. M. A. M. Van Der Horst, and R. P. M. Steegers-Theunissen, "Maternal nutritional status and the risk for orofacial cleft offspring in humans," Journal of Nutrition, vol. 134, no. 11, pp. 3106-3113, 2004.

[13] S. L. Carmichael, G. M. Shaw, C. Ma, M. M. Werler, S, A. Rasmussen, and E. J. Lammer, "Maternal corticosteroid use and orofacial clefts," American Journal of Obstetrics and Gynecology, vol. 197, no. 6, pp. 585.e1-585.e7, 2007.

[14] M. Melnick, T. Jaskoll, and H. C. Slavkin, "Corticosteroidinduced cleft palate in mice and $\mathrm{H}-2$ haplotype: maternal and embryonic effects," Immunogenetics, vol. 13, no. 5, pp. 443 450, 1981.

[15] R. Azziz and R. L. Ladda, "Dexamethasone receptor levels in palatal and lung fibroblasts of adult $\mathrm{A} / \mathrm{J}$ and $\mathrm{C} 57 \mathrm{BL} / 6 \mathrm{~J}$ mice: relationship to glucocorticoid-induced cleft palate," Cleft Palate Journal, vol. 27, no. 4, pp. 388-391, 1990.

[16] L. Pinsky and A. M. DiGeorge, "Cleft palate in the mouse: a teratogenic index of glucocorticoid potency," Science, vol. 147, no. 3656, pp. 402-403, 1965.

[17] T. L. Thomae, E. Glover, and C. A. Bradfield, "A maternal Ahr null genotype sensitizes embryos to chemical teratogenesis," Journal of Biological Chemistry, vol. 279, no. 29, pp. 3018930194, 2004.

[18] T. Hamachi, Y. Sasaki, K. Hidaka, and M. Nakata, "Association between palatal morphosenesis and Pax9 expression pattern in CL/Fr embryos with clefting during palatal development," Archives of Oral Biology, vol. 48, no. 8, pp. 581-587, 2003.

[19] T. Senda, N. Natsume, J. Kuno, T. Toyoda, and K. Shimozato, "Rate of occurrence of dexamethasone-induced cleft palate affected by uterine environment in the mouse," Plastic and Reconstructive Surgery, vol. 115, no. 4, pp. 1208-1210, 2005.
[20] O. Fiehn, "Metabolomics - the link between genotypes and phenotypes," Plant Molecular Biology, vol. 48, no. 1-2, pp. 155171, 2002.

[21] B. C. M. Potts, A. J. Deese, G. J. Stevens, M. D. Reily, D. G. Robertson, and J. Theiss, "NMR of biofluids and pattern recognition: assessing the impact of NMR parameters on the principal component analysis of urine from rat and mouse," Journal of Pharmaceutical and Biomedical Analysis, vol. 26, no. 3, pp. 463-476, 2001.

[22] J. L. Griffin, H. J. Williams, E. Sang, K. Clarke, C. Rae, and J. K. Nicholson, "Metabolic profiling of genetic disorders: a multitissue ${ }^{1} \mathrm{H}$ nuclear magnetic resonance spectroscopic and pattern recognition study into dystrophic tissue," Analytical Biochemistry, vol. 293, no. 1, pp. 16-21, 2001.

[23] J. T. Brindle, H. Antti, E. Holmes et al., "Rapid and noninvasive diagnosis of the presence and severity of coronary heart disease using ${ }^{1} \mathrm{H}-\mathrm{NMR}$-based metabonomics," Nature Medicine, vol. 8, no. 12, pp. 1439-1444, 2002.

[24] M. E. Bollard, E. Holmes, J. C. Lindon et al., "Investigations into biochemical changes due to diurnal variation and estrus cycle in female rats using high-resolution ${ }^{1} \mathrm{H}-\mathrm{NMR}$ spectroscopy of urine and pattern recognition," Analytical Biochemistry, vol. 295, no. 2, pp. 194-202, 2001.

[25] N. J. Waters, E. Holmes, C. J. Waterfield, R. D. Farrant, and J. K. Nicholson, "NMR and pattern recognition studies on liver extracts and intact livers from rats treated with $\alpha$ naphthylisothiocyanate," Biochemical Pharmacology, vol. 64, no. 1, pp. 67-77, 2002.

[26] E. Holmes and H. Antti, "Chemometric contributions to the evolution of metabonomics: mathematical solutions to characterising and interpreting complex biological NMR spectra," Analyst, vol. 127, no. 12, pp. 1549-1557, 2002.

[27] J. Taylor, R. D. King, T. Altmann, and O. Fiehn, "Application of metabolomics to plant genotype discrimination using statistics and machine learning," Bioinformatics, vol. 18, supplement 2, pp. S241-S248, 2002.

[28] J. K. Nicholson, J. C. Lindon, and E. Holmes, "'Metabonomics': understanding the metabolic responses of living systems to pathophysiological stimuli via multivariate statistical analysis of biological NMR spectroscopic data," Xenobiotica, vol. 29, no. 11, pp. 1181-1189, 1999.

[29] J. K. Nicholson, J. Connelly, J. C. Lindon, and E. Holmes, "Metabonomics: a platform for studying drug toxicity and gene function," Nature Reviews Drug Discovery, vol. 1, no. 2, pp. 153-161, 2002.

[30] J. C. Lindon, E. Holmes, and J. K. Nicholson, "Pattern recognition methods and applications in biomedical magnetic resonance," Progress in Nuclear Magnetic Resonance Spectroscopy, vol. 39, no. 1, pp. 1-40, 2001.

[31] R. M. Greene and D. M. Kochhar, "Some aspects of corticosteroid induced cleft palate: a review," Teratology, vol. 11, no. 1, pp. 47-55, 1975.

[32] J. F. Hackney, "A glucocorticoid receptor in fetal mouse: its relationship to cleft palate formation," Teratology, vol. 21, no. 1, pp. 39-51, 1980.

[33] R. M. Pratt, E. L. Perry, L. M. Chapman, and E. H. Goulding, "Glucocorticoid teratogenesis in mouse whole embryo culture," Teratology, vol. 30, no. 1, pp. 71-81, 1984.

[34] M. Melnick, T. Jaskoll, and H. C. Slavkin, "Corticosteroidinduced cleft palate in mice and $\mathrm{H}-2$ haplotype: maternal and embryonic effects," Immunogenetics, vol. 13, no. 5, pp. 443450, 1981.

[35] Y. Eishi, K. Hirokawa, and S. Hatakeyama, "Long-lasting impairment of immune and endocrine systems of offspring 
induced by injection of dexamethasone into pregnant mice," Clinical Immunology and Immunopathology, vol. 26, no. 3, pp. 335-349, 1983.

[36] H. Tang, Y. Wang, J. K. Nicholson, and J. C. Lindon, "Use of relaxation-edited one-dimensional and two dimensional nuclear magnetic resonance spectroscopy to improve detection of small metabolites in blood plasma," Analytical Biochemistry, vol. 325, no. 2, pp. 260-272, 2004.

[37] E. Holmes, A. W. Nicholls, J. C. Lindon et al., "Development of a model for classification of toxin-induced lesions using ${ }^{1} \mathrm{H}$ NMR spectroscopy of urine combined with pattern recognition," NMR in Biomedicine, vol. 11, no. 4-5, pp. 235-244, 1998.

[38] E. Holmes, A. W. Nicholls, J. C. Lindon et al., "Chemometric models for toxicity classification based on NMR spectra of biofluids," Chemical Research in Toxicology, vol. 13, no. 6, pp. 471-478, 2000.

[39] D. Coomans, I. Broeckaert, M. P. Derde, A. Tassin, D. L. Massart, and S. Wold, "Use of microcomputer for the definition of multivariate confidence regions in medical diagnosis based on clinical laboratory profiles," Computers and Biomedical Research, vol. 17, no. 1, pp. 1-14, 1984.

[40] M. E. Bollard, E. G. Stanley, J. C. Lindon, J. K. Nicholson, and E. Holmes, "NMR-based metabonomic approaches for evaluating physiological influences on biofluid composition," NMR in Biomedicine, vol. 18, no. 3, pp. 143-162, 2005.

[41] F. X. R. van Leeuwen, Dexamethasone, IPCS INCHEM, http:// www.inchem.org/documents/jecfa/jecmono/v33je09.htm.

[42] R. Azziz and R. L. Ladda, "Dexamethasone receptor levels in palatal and lung fibroblasts of adult $\mathrm{A} / \mathrm{J}$ and $\mathrm{C} 57 \mathrm{BL} / 6 \mathrm{~J}$ mice: relationship to glucocorticoid-induced cleft palate," Cleft Palate Journal, vol. 27, no. 4, pp. 388-392, 1990.

[43] I. A. L. M. Van Rooij, D. W. Swinkels, H. J. Blom, H. M. W. M. Merkus, and R. P. M. Steegers-Theunissen, "Vitamin and homocysteine status of mothers and infants and the risk of nonsyndromic orofacial clefts," American Journal of Obstetrics and Gynecology, vol. 189, no. 4, pp. 1155-1160, 2003.

[44] M. Vujkovic, M. C. Ocke, P. J. Van Der Spek, N. Yazdanpanah, E. A. Steegers, and R. P. Steegers-Theunissen, "Maternal western dietary patterns and the risk of developing a cleft lip with or without a cleft palate," Obstetrics and Gynecology, vol. 110, no. 2, pp. 378-384, 2007.

[45] L. S. Kerzner, B. S. Stonestreet, K.-Y. Wu, G. Sadowska, and M. P. Malee, "Antenatal dexamethasone: effect on ovine placental $11 \beta$-hydroxysteroid dehydrogenase type 2 expression and fetal growth," Pediatric Research, vol. 52, no. 5, pp. 706-712, 2002.

[46] M. E. Bollard, H. C. Keun, O. Beckonert et al., "Comparative metabonomics of differential hydrazine toxicity in the rat and mouse," Toxicology and Applied Pharmacology, vol. 204, no. 2, pp. 135-151, 2005.

[47] S. Vangala and A. Tonelli, "Biomarkers, metabonomics, and drug development: can inborn errors of metabolism help in understanding drug toxicity?" AAPS Journal, vol. 9, no. 3, pp. E284-E297, 2007.

[48] D. H. Baker and G. L. Czarnecki, "Transmethylation of homocysteine to methionine: efficiency in the rat and chick," Journal of Nutrition, vol. 115, no. 10, pp. 1291-1299, 1985. 

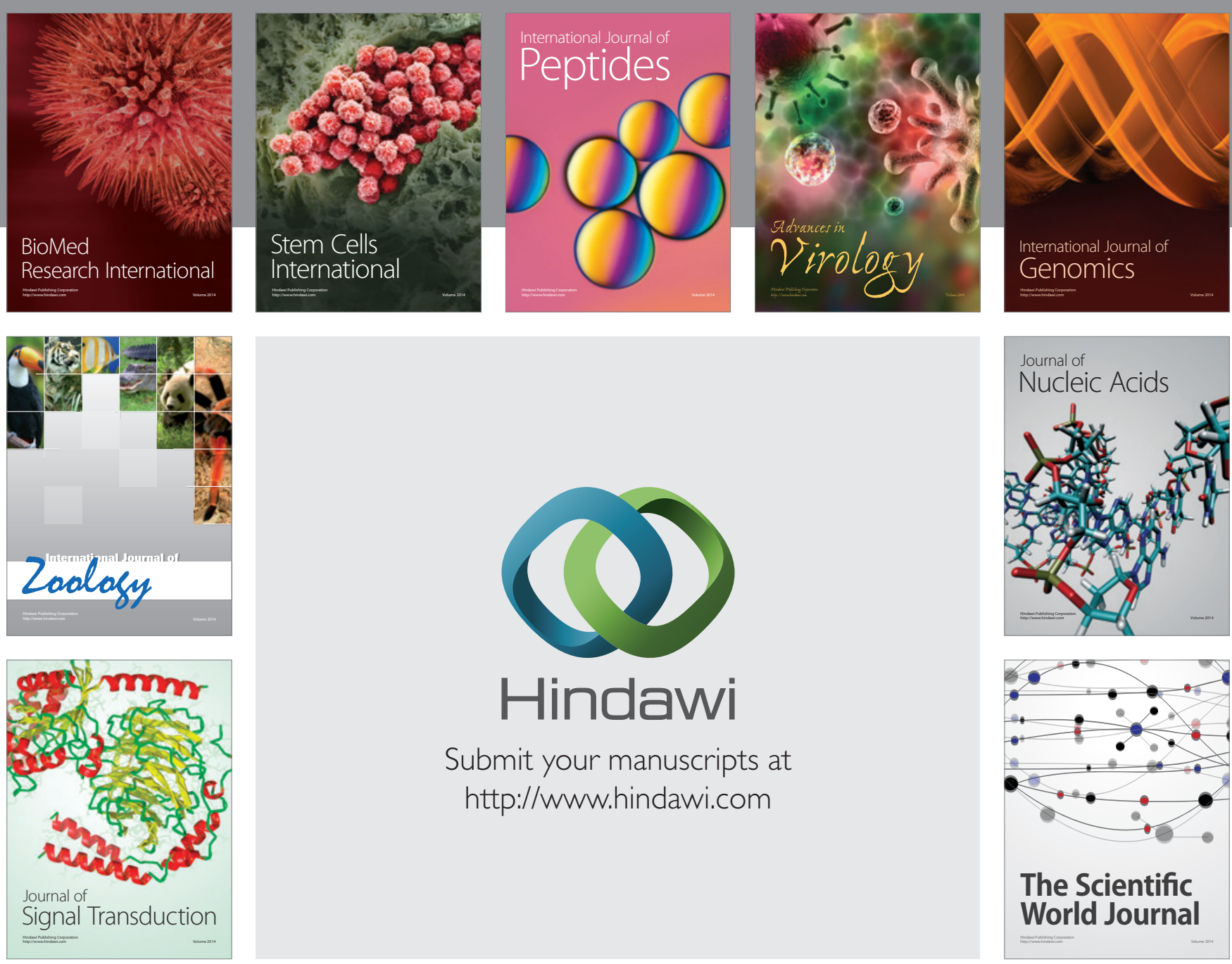

Submit your manuscripts at

http://www.hindawi.com
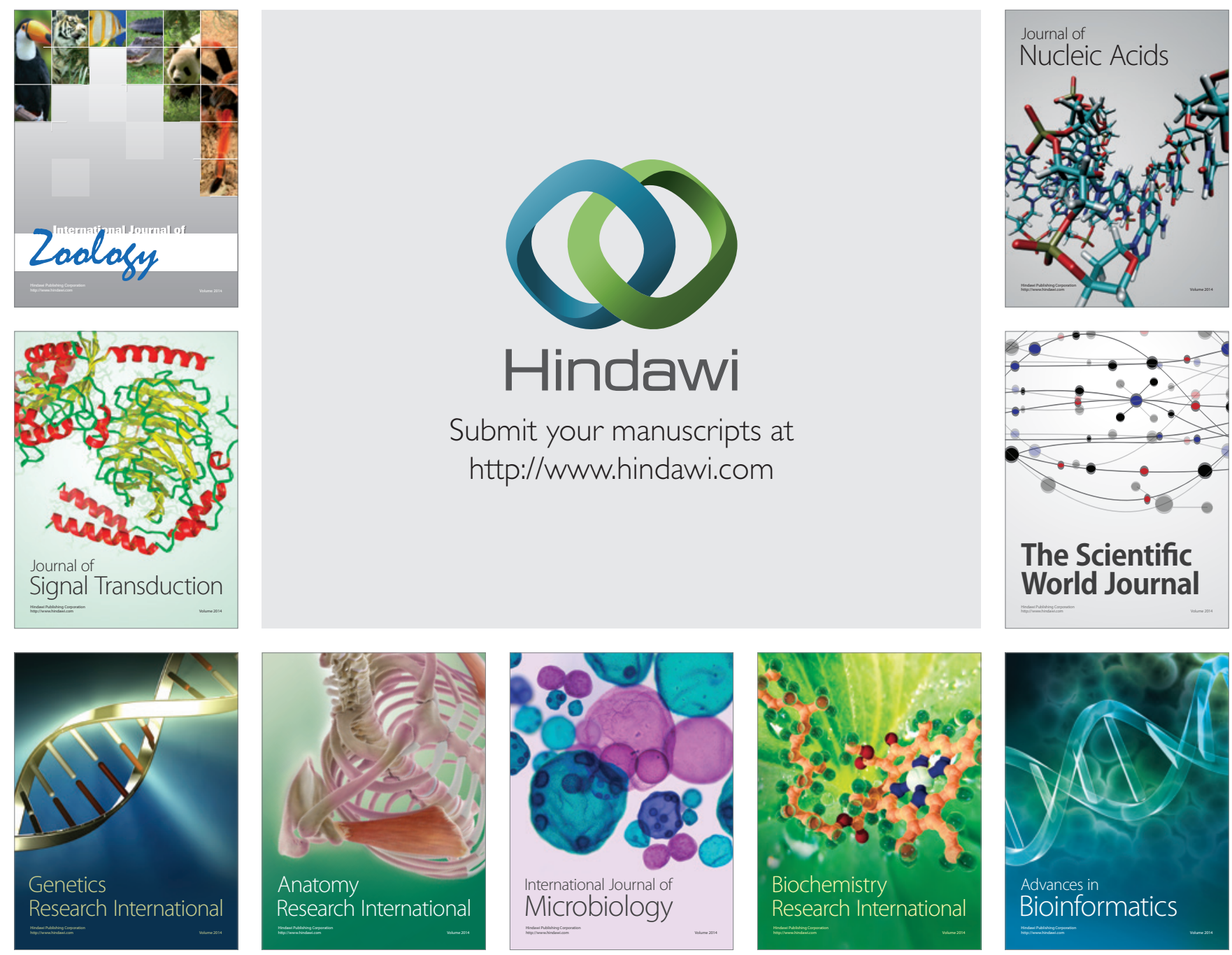

The Scientific World Journal
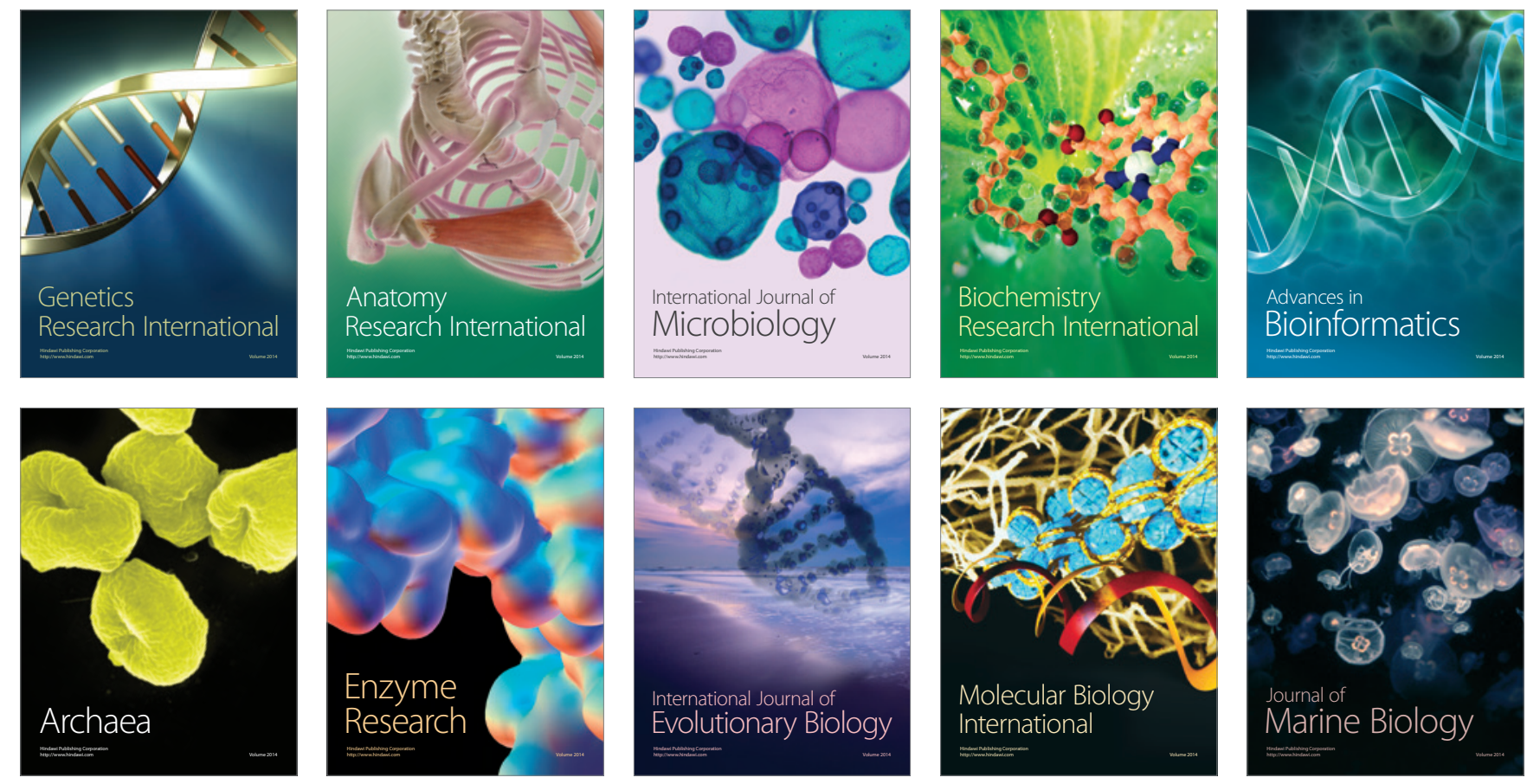